\section{Mortalidade por câncer em populações indígenas no Estado do Acre, Brasil}

\author{
Cancer mortality among indigenous population in \\ Acre State, Brazil
}

\section{Mortalidad por cáncer en poblaciones indígenas en el estado de Acre, Brasil}

Maria Fernanda de Sousa Oliveira Borges 1

Sergio Koifman $†$

Rosalina Jorge Koifman 2

Ilce Ferreira da Silva 2

\section{Resumo}

O objetivo do estudo foi estimar a mortalidade por câncer em povos indígenas no Estado do Acre, Brasil. Trata-se de estudo observacional descritivo, com base no banco nominal do SIM (Sistema de Informações sobre Mortalidade), referente ao período de 1o de janeiro de 2000 a 31 de dezembro de 2012. Foi analisada a distribuição de frequência de óbitos, por sexo e faixa etária, e calculada a RMP (razão de mortalidade padronizada), tendo como referência Goiânia (Goiás), Acre e Região Norte. Foram identificados 81 óbitos, a maioria de homens (59,3\%) e acima de 70 anos. As cinco principais localizações em homens foram estômago, fígado, cólon e reto, leucemia e próstata. Nas mulheres, câncer cervical, estômago, fígado, leucemia e útero. Nos homens indígenas houve excesso de óbitos por câncer de estômago quando comparados às populações de Goiânia $(R M P=2,72 ; 2,58-2,87)$, Acre $(R M P=2,05 ; 1,94-2,16) e$ Região Norte $(R M P=3,10 ; 2,93-3,27)$. O mesmo foi observado para óbitos por hepatocarcinomas, tendo por referência Goiânia $(R M P=3,89 ; 3,66-4,14)$, Acre $(R M P=1,79 ; 1,68-1,91)$ e Região Norte $(R M P=4,04 ; 3,77-4,30)$. Dentre as mulheres indigenas, destaca-se o excesso de câncer cervical em relação à Goiânia $(R M P=4,67 ; 4,41-4,93)$, Acre $(R M P=2,12 ; 2,00-2,24)$ e Região Norte $(R M P=2,60 ; 2,45-2,75)$. As estimativas apontam que neoplasias passíveis de prevenção, como câncer cervical, e ligadas ao subdesenvolvimento, como estômago e fígado, corresponderam a cerca de 49,4\% dos óbitos entre indígenas. Comparados à população de referência, a mortalidade por câncer de fígado, estômago, colorretal e leucemias foi maior que o dobro entre os homens indígenas; por câncer cervical, estômago, fígado e leucemias esteve acima de $30 \%$ entre as mulheres indígenas.

Saúde de Populações Indígenas; Neoplasias; Mortalidade

\author{
Correspondência \\ M. F. S. O. Borges \\ Universidade Federal do Acre. \\ Rua W1, 136, Rio Branco, AC 69919-775, Brasil. \\ fernandacomjesus@hotmail.com \\ 1 Universidade Federal do Acre, Rio Branco, Brasil. \\ 2 Escola Nacional de Saúde Pública Sergio Arouca, Fundação \\ Oswaldo Cruz, Rio de Janeiro, Brasil. \\ $\uparrow$ Falecido.
}




\section{Introdução}

Estima-se que existam cerca de 370 milhões de indígenas distribuídos em 90 países ao redor do mundo, representando aproximadamente 5 mil culturas e 7 mil línguas distintas. Equivalendo a 5\% da população mundial, os povos indígenas compõem 15\% dos mais pobres e compartilham problemas comuns relacionados a precárias condições sociais, econômicas, de educação e saúde, quando comparados às contrapartes não indígenas 1 .

No Brasil, embora representem apenas 0,4\% da população nacional (896,9 mil pessoas), os povos indígenas apresentam uma das maiores diversidades do mundo, correspondendo a 305 etnias e 274 línguas, com maior contingente na Região Norte $(38,2 \%)^{2}$.

$\mathrm{Na}$ Amazônia, os povos indígenas experimentam uma trajetória histórica de subordinação e desvantagem socioeconômica, num cenário de iniquidades que são traduzidas em piores indicadores de saúde, como alta mortalidade infantil, elevadas prevalências de desnutrição infantil, maiores taxas de morbidade e mortalidade por doenças infecto-parasitárias 3,4,5,6,7,8,9. Entretanto, embora as doenças transmissíveis permaneçam como as principais causas de morbimortalidade indígena, observa-se o crescimento dos agravos externos e doenças crônicas não transmissíveis, com destaque para obesidade, hipertensão arterial, diabetes e neoplasias, no contexto de transição epidemiológica vivenciado por esses povos nas últimas décadas 10,11,12,13.

Em relação à mortalidade, há escassez de estudos para a população indígena do país. Estimativas baseadas nos dados de óbitos do Censo Demográfico de 2010 apontam maiores taxas entre indígenas do que entre não indígenas, em todos os grupos etários e em ambos os sexos, sobretudo em mulheres e crianças 14 . Em se tratando da mortalidade por câncer não existem dados com representatividade nacional e poucos estudos demográficos foram realizados em etnias específicas $15,16,17,18$. Todavia, as neoplasias representam baixa proporção das causas de óbitos em indígenas, que apresentam maior carga de mortalidade na infância e por doenças infecciosas 5,14 .

Embora exista um estudo sobre mortalidade por neoplasias na população da capital do Acre, tais investigações inexistem para os povos indígenas do estado ${ }^{19}$. Diante disso, o objetivo deste trabalho consiste em estimar a mortalidade por câncer em povos indígenas no Estado do Acre, a fim de contribuir com o conhecimento epidemiológico das neoplasias nesta população e no planejamento dos serviços de saúde.

\section{Métodos}

Trata-se de estudo descritivo, com uso de dados secundários obtidos do banco nominal do Sistema de Informações sobre Mortalidade (SIM), da Secretaria Estadual de Saúde do Acre, referentes ao período de 1o de janeiro de 2000 a 31 de dezembro de 2012. A identificação dos óbitos indígenas ocorreu com base no item raça/cor, que classifica o indivíduo em uma das cinco categorias: branca, preta, amarela, parda ou indígena. Além disso, o acesso ao banco nominal foi realizado para garantir maior identificação diante da possibilidade de subnotificação. Considerando que entre indígenas o sobrenome contempla a filiação étnica, a abordagem nominal tem sido utilizada com êxito em estudos com o uso de banco de dados 20,21.

A análise descritiva da distribuição de frequências de câncer foi realizada segundo sexo e faixa etária, usando-se os códigos das neoplasias da Classificação Internacional de Doenças, 10a revisão (CID-10).

A razão de mortalidade padronizada (RMP) foi a medida de associação utilizada, sendo calculada por meio da razão entre óbitos observados pelo somatório dos óbitos esperados se a população de estudo sofresse as mesmas magnitudes das taxas de mortalidade específicas por grupo etário da população adotada como referência. Essa medida de associação descritiva dentro de uma coorte requer desagregação por sexo e idade, permitindo o cálculo de pessoa-tempo de cada estrato etário da população de estudo. O cálculo de pessoa-tempo populacional é realizado usando-se o produto do tamanho médio da população durante o período, pela duração do período a risco 22 . Assim, o cálculo de pessoa-tempo da população indígena foi efetuado baseando-se na média da população entre os censos de 2000 e 2010, segundo sexo e estratos etários, seguida da multiplicação pelos anos analisados (13 anos). 
As populações de referência foram compostas por Goiânia (Goiás), Acre e Região Norte, com taxas de mortalidade obtidas dos dados oficiais (https://mortalidade.inca.gov.br/MortalidadeWeb/).

A cidade de Goiânia, localizada na Região Centro-oeste, foi escolhida por ser referência histórica no tratamento de neoplasias para a população do Acre, principalmente até 2006, período em que inexistia hospital oncológico no estado. Os Registros de Câncer de Goiânia apresentam alto grau de acurácia das informações, favorecendo a capacidade de determinar incidência e mortalidade 23. O Registro de Câncer de Base Populacional (RCBP) de Goiânia atua de acordo com critérios de qualidade preconizados pela Agência Internacional de Pesquisa em Câncer (IARC), sendo um dos poucos registros do Brasil utilizado nas publicações desta agência 24. As taxas de mortalidade de Goiânia para cálculo do número de óbitos esperados foram de 2006, que corresponde à metade do período estudado, estratégia frequentemente adotada.

Entretanto, em relação ao Estado do Acre e Região Norte optou-se por utilizar as taxas de mortalidade de 2012, devido à melhoria na acurácia dos dados com o passar dos anos, podendo não estar retratada a real magnitude das taxas de mortalidade caso se adotasse o ano de 2006. Na Região Norte, o índice de adequação das informações de óbitos do SIM aumentou de 46,5 (1999-2001) para 79,6\% (2008-2010), com taxa anual de variação de 7,2\%. No Acre, o aumento foi de 35\% (1999-2001) para 69,9\% (2008-2010), indicando melhoria na qualidade dos dados ao longo do tempo em relação à cobertura, regularidade do sistema e qualidade das notificações dos óbitos relativos à causa básica 25 .

Para as estimativas do intervalo de 95\% de confiança (IC95\%), considerando-se o pressuposto de que os valores amostrais da RMP são normalmente distribuídos, a variância foi estimada com base nos dados da amostra e na equação: RMP $\pm 1.96(\mathrm{RMP} / \mathrm{E}) 1 / 2=\mathrm{RMP} 26$.

Para a elaboração das tabelas foi utilizado o programa Excel (https://products.office.com/) e para a análise dos dados foi utilizado o aplicativo SPSS para Windows (versão 22.0; https://www.ibm.com/).

O presente estudo foi aprovado pelo Comitê de Ética em Pesquisa (CEP) da Universidade Federal do Acre (UFAC) e da Escola Nacional de Saúde Pública Sergio Arouca, Fundação Oswaldo Cruz (ENSP/Fiocruz), após anuência da Comissão Nacional de Ética em Pesquisa (CONEP), sendo financiado pelo Programa de Pesquisa para o SUS (PPSUS), Edital 001/2013 da Fundação de Amparo à Pesquisa do Acre (FAPAC).

\section{Resultados}

Foram identificados 81 óbitos por neoplasias (CID-10: C00-C97) em indígenas no Acre, dos quais, 9 $(11,1 \%)$ foram reclassificados com base na identificação nominal. A maioria do sexo masculino (59,3\%) e na faixa etária acima de 70 anos. Entre as etnias, a que apresentou maior frequência foi o povo Huni Kuin/Kaxinawá (38,8\%), a maior do estado (Tabela 1).

A Tabela 2 apresenta a média da população indígena por sexo e idade, dos censos de 2000 e 2010, expressando o tempo de contribuição de cada faixa etária, o cálculo de pessoa-ano referente ao seguimento do estudo, bem como a frequência dos óbitos segundo sexo e faixa etária.

$\mathrm{Na}$ Tabela 3, identificam-se os óbitos por sexo segundo topografia e código da neoplasia (CID-10). As cinco principais causas de óbitos por câncer em homens foram estômago (25,5\%), fígado (19,1\%), cólon e reto $(8,5 \%)$, leucemia $(8,5 \%)$ e próstata $(8,5 \%)$. Entre as mulheres foram câncer do colo do útero $(36,4 \%)$, estômago $(12,1 \%)$, fígado $(6,1 \%)$, leucemia $(6,1 \%)$, neoplasia secundária de órgãos digestivos $(6,1 \%)$ e útero $(6,1 \%)$.

Entre os homens indígenas (Tabela 4), a mortalidade para todas as localizações de câncer foi menor do que a esperada se a população do estudo tivesse as mesmas taxas de mortalidade dos homens de Goiânia (RMP = 0,73; IC95\%: 0,71-0,75). Contudo, verifica-se um excesso de óbitos entre indígenas por câncer de fígado (RMP = 3,89; IC95\%: 3,66-4,14) e de estômago (RMP = 2,72; IC95\%: 2,58-2,87). Destaca-se que há menor mortalidade para o câncer de próstata $(R M P=0,52$; IC95\%: 0,43-0,62) e leucemia (RMP = 0,85; IC95\%: 0,77-0,94).

A mortalidade para todas as localizações foi semelhante à da população geral masculina do Acre $(\mathrm{RMP}=1,03$; IC95\%: 0,99-1,06), sendo cerca de 26\% maior em homens indígenas quando comparados aos homens da Região Norte (RMP = 1,26; IC95\%: 1,23-1,30). Um excesso de óbitos entre homens indígenas foi identificado tanto em comparação com os dados do Acre quanto do Norte para o 
Tabela 1

Distribuição de frequências absolutas e relativas dos óbitos por câncer em populações indígenas, segundo variáveis selecionadas. Acre, Brasil, 2000-2012.

\begin{tabular}{|c|c|c|c|}
\hline Variável & $\begin{array}{c}\text { Homens } \\
\text { n (\%) }\end{array}$ & $\begin{array}{c}\text { Mulheres } \\
\text { n (\%) }\end{array}$ & $\begin{array}{l}\text { Total } \\
\text { n (\%) }\end{array}$ \\
\hline \multicolumn{4}{|l|}{ Faixa etária (anos) } \\
\hline $0-4$ & $1(2,0)$ & $1(3,0)$ & $2(2,5)$ \\
\hline $5-9$ & $2(4,2)$ & $0(0,0)$ & $2(2,5)$ \\
\hline $10-14$ & $2(4,2)$ & $0(0,0)$ & $2(2,5)$ \\
\hline $15-19$ & $2(4,2)$ & $1(3,0)$ & $3(3,7)$ \\
\hline $20-29$ & $1(2,0)$ & $3(9,1)$ & $4(4,9)$ \\
\hline $30-39$ & $2(4,2)$ & $3(9,1)$ & $5(6,2)$ \\
\hline $40-49$ & $2(4,2)$ & $3(9,1)$ & $5(6,2)$ \\
\hline $50-59$ & $8(16,7)$ & $3(9,1)$ & $11(13,5)$ \\
\hline $60-69$ & $13(27,1)$ & $4(12,1)$ & $17(21,0)$ \\
\hline 70 ou mais & $15(31,2)$ & $15(45,5)$ & $30(37,0)$ \\
\hline \multicolumn{4}{|l|}{ Etnia * } \\
\hline Apolima Arara & $0(0,0)$ & $2(6,1)$ & $2(2,5)$ \\
\hline Apurinã & $2(4,2)$ & $4(12,1)$ & $6(7,4)$ \\
\hline Ashaninka & $2(4,2)$ & $0(0,0)$ & $2(2,5)$ \\
\hline Jamamadi & $1(2,1)$ & $2(6,1)$ & $3(3,7)$ \\
\hline Jaminawa & $5(10,4)$ & $1(3,0)$ & $6(7,4)$ \\
\hline Katukina & $0(0,0)$ & $4(12,1)$ & $4(4,9)$ \\
\hline Kaxarari & $0(0,0)$ & $1(3,0)$ & $1(1,2)$ \\
\hline Kaxinawá (Huni Kuin) & $23(47,9)$ & $8(24,2)$ & $31(38,3)$ \\
\hline Kulina & $1(2,1)$ & $1(3,0)$ & $2(2,5)$ \\
\hline Manchineri & $2(4,2)$ & $0(0,0)$ & $2(2,5)$ \\
\hline Nukini & $1(2,1)$ & $0(0,0)$ & $1(1,2)$ \\
\hline Poyanawa & $1(2,1)$ & $1(3,0)$ & $2(2,5)$ \\
\hline Shanenawa & $1(2,1)$ & $0(0,0)$ & $1(2,1)$ \\
\hline Yawanawá & $3(6,3)$ & $0(0,0)$ & $3(3,7)$ \\
\hline Total & $48(100,0)$ & $33(100,0)$ & $81(100,0)$ \\
\hline
\end{tabular}

* Os valores perdidos correspondem a pessoas indígenas cujas etnias não foram identificadas $(n=15)$.

câncer de estômago (RMPAcre = 2,05; IC95\%: 1,94-2,16; RMPNorte = 3,10; IC95\%: 2,93-3,27), fígado $($ RMPAcre = 1,79; IC95\%: 1,68-1,91; RMPNorte = 4,04; IC95\%: 3,77-4,30), cólon e reto (RMPAcre = 5,06; IC95\%: 4,57-5,56; RMPNorte = 3,36; IC95\%: 3,19-3,53), leucemias (RMPAcre = 2,37; IC95\%: 2,13-2,60); RMPNorte = 1,88; IC95\%: 1,69-2,06) e neoplasia maligna sem especificação da localização (RMPAcre = 1,61; IC95\%: 1,43-1,80; RMPNorte = 1,32; IC95\%: 1,17-1,47). Porém, menor mortalidade foi observada para o câncer de próstata (RMPAcre = 0,52; IC95\%: 0,47-0,57; RMPNorte = 0,70; IC95\%: 0,63-0,77) (Tabela 4).

Nas mulheres indígenas também verificou-se menor mortalidade para todas as localizações de câncer em relação às mulheres de Goiânia (RMP =0,79; IC95\%: 0,76-0,82). Porém, verifica-se um excesso de óbitos em indígenas para o câncer do colo do útero (RMP = 4,67; IC95\%: 4,41-4,93), fígado (RMP = 1,87; IC95\%: 1,61-2,12) e estômago (RMP = 1,57; IC95\%: 1,42-1,73). Em contraste, a mortalidade por câncer de mama $(\mathrm{RMP}=0,21$; IC95\%: 0,16-0,25) e leucemias $(\mathrm{RMP}=0,63$; IC95\%: 0,54-0,72) foi menor (Tabela 5).

Em relação ao Estado do Acre e Região Norte, observa-se que a mortalidade para todas as localizações em mulheres indígenas foi semelhante ao esperado (RMPAcre = 1,02; IC95\%: 0,99-1,06; 


\section{Tabela 2}

População indígena, segundo sexo e faixa etária, dos censos de 2000 e 2010, dados de pessoa-ano e óbitos identificados no período $2000-2012$. Acre, Brasil.

\begin{tabular}{|c|c|c|c|c|c|c|}
\hline \multirow[t]{2}{*}{ Idade (anos) } & \multicolumn{2}{|c|}{$\begin{array}{c}\text { Média populacional dos } \\
\text { indígenas por sexo } \\
\text { (Censos Demográficos de } 2000 \text { e 2010) }\end{array}$} & \multicolumn{2}{|c|}{$\begin{array}{l}\text { Pessoa-ano } \\
(2000-2012)\end{array}$} & \multicolumn{2}{|c|}{$\begin{array}{l}\text { Óbitos por câncer observados } \\
\text { (2000-2012) }\end{array}$} \\
\hline & Homens & Mulheres & Homens & Mulheres & Homens & Mulheres \\
\hline Total & 6.488 & 6.306 & $84.337,50$ & $81.978,00$ & 48 & 33 \\
\hline $0-4$ & 1.271 & 1.251 & $16.528,29$ & $16.261,41$ & 1 & 1 \\
\hline $5-9$ & 1.063 & 1.043 & $13.813,13$ & $13.558,44$ & 2 & 0 \\
\hline $10-14$ & 871 & 800 & $11.318,13$ & $10.399,59$ & 2 & 0 \\
\hline $15-19$ & 666 & 657 & $8.657,78$ & $8.538,29$ & 2 & 1 \\
\hline $20-29$ & 1.027 & 983 & $13.351,00$ & $12.779,00$ & 1 & 3 \\
\hline $30-39$ & 668 & 632 & $8.682,21$ & $8.219,15$ & 2 & 3 \\
\hline $40-49$ & 495 & 337 & $6.429,16$ & $4.379,84$ & 2 & 3 \\
\hline $50-59$ & 221 & 238 & $2.872,99$ & $3.087,85$ & 8 & 3 \\
\hline $60-69$ & 194 & 214 & $2.521,56$ & $2.783,63$ & 13 & 4 \\
\hline 70 ou mais & 144 & 151 & $1.871,28$ & $1.958,97$ & 15 & 15 \\
\hline
\end{tabular}

\section{Tabela 3}

Distribuição de frequências de óbitos por câncer em homens e mulheres indígenas, segundo topografia (CID-10). Acre, Brasil, 2000-2012.

\begin{tabular}{lccc}
\hline Topografia/(CID-10) & Homens (\%) & Mulheres (\%) & Total (\%) \\
\hline Todas as localizações (C00-97) & $48(100,0)$ & $33(100,0)$ & $81(100,0)$ \\
Estômago (C16) & $13(27,1)$ & $4(12,1)$ & $17(21,0)$ \\
Colo do útero (C53) & - & $12(36,4)$ & $12(14,8)$ \\
Fígado (C22) & $9(18,7)$ & $2(6,1)$ & $11(13,6)$ \\
Leucemia (C91-95) & $4(8,3)$ & $2(6,1)$ & $6(7,4)$ \\
Cólon e reto (C18, 20) & $4(8,3)$ & $1(3,0)$ & $5(6,2)$ \\
Próstata (C61) & $4(8,3)$ & - & $4(4,9)$ \\
Neoplasia maligna sem especificação da localização (C80) & $3(6,2)$ & $1(3,0)$ & $4(4,9)$ \\
Pele (C44) & $2(4,2)$ & - & $2(2,5)$ \\
Útero (C55) & - & $2(6,1)$ & $2(2,5)$ \\
Rim (C64) & $1(2,1)$ & $1(3,0)$ & $2(2,5)$ \\
Encéfalo e meninges (C71, 79.3) & $2(4,2)$ & - & $2(2,5)$ \\
Neoplasia secundária de órgãos digestivos (C78.8) & - & $2(6,1)$ & $2(2,5)$ \\
Intestino (C17) & $1(2,1)$ & - & $1(1,2)$ \\
Vias Biliares (C24) & $1(2,1)$ & - & $1(1,2)$ \\
Pâncreas (C25) & $1(2,1)$ & - & $1(1,2)$ \\
Neoplasia de seio maxilar (C31) & - & $1(3,0)$ & $1(1,2)$ \\
Traqueia, brônquios e pulmão (C34) & - & $1(3,0)$ & $1(1,2)$ \\
Tumor de células gigantes (C40) & $1(2,1)$ & - & $1(1,2)$ \\
Mama (C50) & - & $1(3,0)$ & $1(1,2)$ \\
Bexiga (C67) & $1(2,1)$ & - & $1(1,2)$ \\
Glândula parauretral (C68) & $1(2,1)$ & - & $1(1,2)$ \\
Olhos e anexos (C69) & - & $1(3,0)$ & $1(1,2)$ \\
Neoplasia maligna secundária do mediastino (C78.1) & - & $1(1,2)$ \\
Neoplasia secundária do peritônio e retroperitônio (C78.6) & - & $1(1,2)$ \\
\hline
\end{tabular}

CID-10: Classificação Internacional de Doenças, 10ạ revisão. 


\section{Tabela 4}

Mortalidade observada e esperada por todas as localizações anatômicas de câncer e localizações selecionadas em homens indígenas do Acre, Brasil, 2000-2012.

\begin{tabular}{|c|c|c|c|c|c|c|c|}
\hline \multirow[t]{2}{*}{ Localização anatômica de câncer (CID-10) } & \multirow[t]{2}{*}{0} & \multicolumn{2}{|c|}{ População Goiânia * } & \multicolumn{2}{|c|}{ População Acre ** } & \multicolumn{2}{|c|}{ População Região Norte *** } \\
\hline & & E & $\begin{array}{l}\text { RMP } \\
\text { (IC95\%) }\end{array}$ & $\mathbf{E}$ & $\begin{array}{l}\text { RMP } \\
\text { (IC95\%) }\end{array}$ & $\mathbf{E}$ & $\begin{array}{l}\text { RMP } \\
\text { (IC95\%) }\end{array}$ \\
\hline Todas as localizações (C00-97) \# & 46 & 61,7 & $0,73(0,71-0,75)$ & 45,0 & $1,03(0,99-1,06)$ & 42,5 & $1,26(1,23-1,30)$ \\
\hline Estômago (C16) & 13 & 4,8 & $2,72(2,58-2,87)$ & 9,7 & $2,05(1,94-2,16)$ & 8,2 & $3,10(2,93-3,27)$ \\
\hline Fígado (C22) & 9 & 2,3 & $3,89(3,66-4,14)$ & 5,0 & $1,79(1,68-1,91)$ & 2,2 & $4,04(3,77-4,30)$ \\
\hline Cólon e reto (C18) & 4 & 3,6 & $1,12(0,98-1,25)$ & 0,8 & $5,06(4,57-5,56)$ & 1,2 & $3,36(3,19-3,53)$ \\
\hline Próstata (C61) & 4 & 7,6 & $0,52(0,43-0,62)$ & 7,7 & $0,52(0,47-0,57)$ & 5,7 & $0,70(0,63-0,77)$ \\
\hline Leucemia (C91-95) & 4 & 4,7 & $0,85(0,77-0,94)$ & 1,7 & $2,37(2,13-2,60)$ & 2,1 & $1,88(1,69-2,06)$ \\
\hline $\begin{array}{l}\text { Neoplasia maligna sem especificação de } \\
\text { local (C80) }\end{array}$ & 3 & 3 & $1,00(0,89-1,11)$ & 1,8 & $1,61(1,43-1,80)$ & 2,3 & $1,32(1,17-1,47)$ \\
\hline
\end{tabular}

CID-10: Classificação Internacional de Doenças, 10ạ revisão; E: óbitos esperados; IC95\%: intervalo de 95\% de confiança; O: óbitos observados; RMP: razão de mortalidade padronizada.

* População de Goiânia - Sistema de Informações de Mortalidade, Departamento de Informática do SUS (2006);

** População do Estado do Acre - Sistema de Informações de Mortalidade, Departamento de Informática do SUS (2012);

*** População da Região Norte - Sistema de Informações de Mortalidade, Departamento de Informática do SUS (2012);

\# Excetuando casos de câncer de pele não melanoma.

\section{Tabela 5}

Mortalidade observada e esperada por todas as localizações anatômicas de câncer e localizações selecionadas em mulheres indígenas do Acre, Brasil, 2000-2012.

\begin{tabular}{|c|c|c|c|c|c|c|c|}
\hline \multirow[t]{2}{*}{ Localização anatômica de câncer (CID-10) } & \multirow[t]{2}{*}{0} & \multicolumn{2}{|c|}{ População Goiânia * } & \multicolumn{2}{|c|}{ População Acre ** } & \multicolumn{2}{|c|}{ População Norte *** } \\
\hline & & E & $\begin{array}{c}\text { RMP } \\
\text { (IC95\%) }\end{array}$ & $\mathbf{E}$ & $\begin{array}{c}\text { RMP } \\
\text { (IC95\%) }\end{array}$ & E & $\begin{array}{c}\text { RMP } \\
\text { (IC95\%) }\end{array}$ \\
\hline Todas as localizações (C00-97) \# & 33 & 41,8 & $0,79(0,76-0,82)$ & 32,3 & $1,02(0,99-1,06)$ & 31,0 & $1,06(1,03-1,10)$ \\
\hline Colo do útero (C53) & 12 & 2,6 & $4,67(4,41-4,93)$ & 5,6 & $2,12(2,00-2,24)$ & 4,6 & $2,60(2,45-2,75)$ \\
\hline Estômago (C16) & 4 & 2,5 & $1,57(1,42-1,73)$ & 1,9 & $2,14(1,93-2,35)$ & 2,4 & $1,64(1,48-1,80)$ \\
\hline Fígado (C22) & 2 & 1,1 & $1,87(1,61-2,12)$ & 2,7 & $0,75(0,65-0,86)$ & 1,5 & $1,36(1,17-1,55)$ \\
\hline Leucemia (C91-95) & 2 & 3,2 & $0,63(0,54-0,72)$ & 1,0 & $1,94(1,67-2,21)$ & 1,9 & $1,07(0,92-1,22)$ \\
\hline Mama (C50) & 1 & 4,8 & $0,21(0,16-0,25)$ & 4,9 & $0,21(0,17-0,25)$ & 3,2 & $0,31(0,25-0,37)$ \\
\hline
\end{tabular}

CID-10: Classificação Internacional de Doenças, 10ª revisão; E: óbitos esperados; IC95\%: intervalo de 95\% de confiança; O: óbitos observados; RMP: razão de mortalidade padronizada.

* População de Goiânia - Sistema de Informações de Mortalidade, Departamento de Informática do SUS (2006);

** População do Estado do Acre - Sistema de Informações de Mortalidade, Departamento de Informática do SUS (2012);

*** População da Região Norte - Sistema de Informações de Mortalidade, Departamento de Informática do SUS (2012);

\# Excetuando casos de câncer de pele não melanoma.

RMPNorte = 1,06; IC95\%: 1,03-1,10). Um excesso de óbitos foi constatado para o câncer do colo do útero (RMPAcre = 2,12; IC95\%: 2,00-2,24; RMPNorte = 2,60; IC95\%: 2,45-2,75) e estômago (RMPAcre = 2,14; IC95\%: 1,93-2,35; RMPNorte = 1,64; IC95\%: 1,48-1,80), bem como para o fígado tendo como referência as mulheres da Região Norte (RMPNorte = 1,36; IC95\%: 1,17-1,55). Todavia, foi menor entre indígenas para o câncer de mama (RMPAcre = 0,21; IC95\%: 0,17-0,25; RMPNorte = 0,31; IC95\%: 0,25-0,37) e fígado (RMPAcre = 0,75; IC95\%: 0,65-0,86) (Tabela 5). 


\section{Discussão}

Neste trabalho, as principais causas de óbito por câncer nos homens indígenas foram estômago, fígado, cólon e reto, leucemia e próstata. Entre as mulheres indígenas foram câncer do colo uterino, estômago, fígado, leucemia, neoplasia secundária de órgãos digestivos e útero. Ressalta-se que câncer de estômago, colo do útero e fígado corresponderam a $49,4 \%$ de todos os óbitos, sendo que a mortalidade nestes sítios foi maior entre indígenas do que a esperada para ambos os sexos, considerando todas as populações de referência, exceto para câncer de fígado nas mulheres indígenas comparadas aos dados do Acre. Esse padrão de mortalidade em indígenas aponta para um perfil epidemiológico com maior relevância das neoplasias relacionadas a agentes infecciosos, situação socioeconômica desfavorável e dificuldades de acesso aos serviços de saúde 27 .

O câncer de estômago foi a segunda causa de morte por esta doença no mundo em ambos os sexos em 2012, apresentando declínio nas taxas ao longo do tempo em diversos países, incluindo o Brasil 28. Tal tendência pode ser atribuída à melhor conservação dos alimentos, redução do uso de sal para acondicionamento e aumento do consumo de alimentos frescos, entre outros fatores que contribuem com a redução da incidência, além da melhoria de acesso ao diagnóstico precoce, que favorece a redução da mortalidade 29 . No Brasil, foi a terceira causa de óbito por câncer em homens e a quinta em mulheres em 2015, com maiores taxas na Região Norte 30. Na capital do Acre, ocupou a quarta posição em ambos os sexos em 2006, sendo verificada nos homens uma tendência decrescente com variação percentual constante de -3,4 ( $p<0,01)$ entre 1980-2006, e uma variação não constante entre as mulheres de $-6,0(\mathrm{p}<0,01)$ e -10,6 ( $<<0,01)$ nos períodos de 1982-1988 e 1995-1998, respectivamente 19.

Na Bolívia, Guatemala e Peru, países com alta proporção de povos indígenas, verifica-se elevada mortalidade por câncer de estômago, alcançando o dobro da média encontrada na América Latina 27. Tal como na presente investigação, em que se observou um excesso de mortalidade por câncer para ambos os sexos, foi identificada maior mortalidade entre indígenas australianos de New South Wales (NSW) (RMPHomens = 2,09; IC95\%: 1,45-2,92; RMPMulheres = 1,98; IC95\%: 1,15-3,16) e entre índios norte-americanos e nativos do Alasca (AI/AN) (MRRHomens $=1,47$ e MRRMulheres $=1,77)$ quando comparados à população geral de seus respectivos territórios 31,32 .

Os estudos indicam uma carga de câncer de estômago desproporcional nos indígenas quando comparados aos não indígenas, favorecida por aspectos comuns à realidade indígena, tais como elevada aglomeração intradomiciliar, inadequada conservação dos alimentos, precariedade na infraestrutura e nas condições de saneamento básico, entre outros, que contribuem para a disseminação da Helicobacter pylori, principal fator associado à etiologia desta neoplasia 27,33,34. Em trabalhos realizados entre indígenas foram encontradas elevadas prevalências dessa bactéria, como entre as crianças do Parque Indígena do Xingu (73,5\%) 35 e da etnia Guarani Mbya (64,3\% entre 4-5 anos e 89,8\% nos pais) 36. Além disso, outros fatores como baixo consumo de vegetais e fibras, tabagismo e abuso de álcool podem contribuir para o desenvolvimento da doença 37 . Tais aspectos, atrelados à dificuldade de acesso aos serviços diagnósticos e tratamento precoce, podem ter implicações importantes para uma maior mortalidade, como a observada na população indígena do Acre.

O câncer do colo do útero é a terceira causa de morte por neoplasias entre mulheres de países menos desenvolvidos, sendo mais raro nos desenvolvidos, onde programas de rastreamento bemsucedidos foram introduzidos há décadas, reduzindo as taxas de incidência e mortalidade 38 . No Brasil, ocupou a quarta posição em mortalidade por câncer em 2015, com padrão diferenciado entre os estados das Regiões Norte (Amapá, Amazonas, Pará e Tocantins) e Nordeste (Maranhão e Piauí), em que sobressaiu em primeiro lugar. No Acre, ocupou a segunda posição entre mulheres em 2010, sendo observada uma tendência de redução da mortalidade entre 1996 e 2010, com variação anual de -6,5\% 30,39. Em Rio Branco, entre 1994 e 2000, foi identificada uma tendência de decréscimo $(-10,7)$ que pode ter sido associada à melhoria no acesso aos serviços de saúde e maior adesão ao rastreamento 19. Porém, o mesmo não pode ser apontado para as mulheres indígenas, já que a estimativa de mortalidade foi cerca de quatro vezes maior que o esperado, tendo como referência as taxas de Goiânia, e cerca de duas vezes maior, considerando as taxas do Acre e da Região Norte. De igual modo, foi observada maior mortalidade entre aborígenes de regiões remotas comparadas a não aborígenes entre 1986-1997 (RMP = 18,3; IC95\%: 14,0-23,9), indígenas australianas de Queensland comparadas 
ao restante das mulheres entre 1997-2006 (RMP = 7,51; IC95\%: 5,45-10,08) e indígenas australianas de NSW entre 1999-2007 (RMP = 4,74; IC95\%: 3,22-6,73) 32,40,41.

Não há dados publicados quanto à cobertura populacional do rastreamento do câncer cervical em mulheres indígenas do Acre. Em comunidades indígenas do país são identificadas elevadas prevalências de lesões precursoras, com frequências que variam de 2,1 a 9,4\% para lesão intraepitelial de baixo grau (LSIL) e de 1,2 a 4,6\% de lesão intraepitelial de alto grau (HSIL), além de serem observados cerca de $2 \%$ de câncer invasor entre mulheres indígenas assintomáticas, superando valores identificados entre mulheres não indígenas 42,43. A elevada mortalidade verificada no presente trabalho pode refletir a dificuldade de acesso às ações de rastreamento, acompanhamento e tratamento adequado das alterações colpocitológicas, aumentando a chance de lesões precursoras evoluírem para neoplasia, diagnóstico em estadiamento avançado e redução da sobrevida 27,44.

Embora a coleta dos exames citopatológicos no SUS seja atribuição da atenção básica, inclusive no Subsistema de Atenção à Saúde Indígena, o tratamento das alterações é realizado na rede de referência, concentrando-se geralmente nos centros urbanos. No Acre, o tratamento ambulatorial das alterações cervicais é realizado desde 1972 no Centro de Controle Oncológico do Acre (CECON), localizado na capital. Porém, até junho de 2007 inexistia hospital oncológico, sendo os casos de câncer enviados para tratamento fora do estado, podendo desfavorecer ainda mais a adesão terapêutica pelas minorias étnicas. Dificuldades relacionadas à desarticulação da rede de referência e contrarreferência dos serviços, isolamento geográfico e barreiras culturais afetam a possibilidade de tratamento oportuno e provocam maior recusa das populações indígenas aos exames e terapias convencionais, gerando piores resultados na mortalidade $45,46,47$.

O câncer de fígado ocupou a segunda e a quarta posições nas estimativas mundiais de mortalidade por neoplasias em homens e mulheres, respectivamente, em 2012 38. Maiores taxas são encontradas no sudeste da Ásia e África Subsaariana, regiões com elevada prevalência do vírus da hepatite B, um dos principais fatores de risco da doença 48. No Brasil, entre 1980 e 2010, observou-se uma tendência de mortalidade crescente para ambos os sexos, sugerindo-se como possíveis causas o elevado número de pessoas infectadas pelas hepatites virais, maior consumo de álcool e acometimento por cirrose hepática, além de melhoria na cobertura e qualidade da informação de mortalidade 49 .

Entre 1980 e 2006, o câncer hepático alcançou a terceira posição em óbitos por câncer em ambos os sexos na capital do Acre ${ }^{19}$. No presente estudo, foi o segundo mais comum em homens e o terceiro em mulheres. Embora exista a possibilidade de ter ocorrido notificação de óbito por metástase hepática como câncer primário, fatores de risco para esta neoplasia, como exposição a aflatoxinas e alta endemicidade das hepatites virais na região, poderiam explicar em parte o excesso significativo de óbitos entre indígenas no Acre 50,51. Semelhantemente, maior mortalidade por hepatocarcinoma foi observada entre indígenas australianos de Queensland entre 1997 e 2006 (RMPHomens = 3,28; IC95\%: 2,29-4,56; RMPMulheres = 2,78; IC95\%: 1,81-4,07) e de NSW entre 1999 e 2007 (RMPhomens = 1,74; IC95\%: 1,06-2,68; RMPmulheres = 2,19; IC95\%: 1,05-4,03) 32,41.

Apesar da baixa frequência, as leucemias também estiveram entre as cinco principais causas de óbitos em ambos os sexos no presente trabalho. As estimativas de RMP dos indígenas do Acre apontaram que, em relação a Goiânia, foi observada menor mortalidade para ambos os sexos. Porém, um excesso foi verificado quando comparados com a população do Acre, e entre homens indígenas comparados aos homens do Norte, podendo refletir a dificuldade de acesso ao diagnóstico e tratamento entre indígenas. A infecção pelo HTLV I/II, a exposição a contaminantes ambientais e a campos eletromagnéticos constituem fatores sugeridos como possíveis causas para o acometimento dessa doença entre povos indígenas 52,53,54.

O câncer colorretal ocupou a terceira e a quarta posições em mortalidade por neoplasias entre mulheres e homens do mundo, respectivamente, em 2012 48. Na população masculina brasileira das regiões Sul e Sudeste foi verificada uma tendência de crescimento entre 1990 e 2015, que pode ser decorrente da associação entre elevados indicadores socioeconômicos e mudanças dos hábitos de vida, bem como pelo aumento do acesso da população-alvo ao rastreamento, elevando o número de diagnósticos 30,55.

Dieta pobre em frutas, verduras e fibras, elevado consumo de carnes e processados, sedentarismo, obesidade, tabagismo e alcoolismo são alguns fatores de risco bem estabelecidos para o desenvolvimento do câncer colorretal 56 . Tais aspectos têm sido observados em diferentes etnias do país, como 
parte do processo de transição epidemiológica, favorecendo que doenças comuns a regiões desenvolvidas sejam identificadas com mais frequência entre indígenas 57 . Câncer colorretal está entre as principais causas de óbito em populações nativas de países desenvolvidos 31,58,59,60,61. Disparidades étnicas traduzidas em diagnóstico tardio e pior sobrevida desse câncer foram observadas entre indígenas em comparação a populações não indígenas $62,63,64,65$. No presente estudo, a maior mortalidade entre homens indígenas, tendo como referência a população masculina do Acre e da Região Norte, sugere menor acesso ao diagnóstico e tratamento precoce da doença.

O câncer de próstata é a quinta causa de mortalidade por neoplasias entre os homens no mundo e a terceira nos países desenvolvidos 28,48. Ocupou o primeiro lugar nos estados brasileiros em 2015, exceto no Amapá, Santa Catarina e Rio Grande do Sul, onde foi a segunda causa de morte por neoplasias 30. Na capital do Acre, verificou-se tendência de crescimento não constante entre $1980 \mathrm{e}$ 2006, alcançando o segundo lugar na mortalidade por câncer entre homens no final deste período. Destaca-se a melhoria das técnicas diagnósticas que podem ter favorecido a detecção, além da maior longevidade 19. No atual trabalho, a mortalidade entre indígenas do Acre foi inferior a de todas as populações utilizadas como referência. Esses resultados podem refletir tanto a menor incidência quanto o baixo acesso aos exames de rastreio pela população-alvo. Em contrapartida, entre indígenas de países desenvolvidos as taxas de incidência são inferiores se comparadas a não indígenas, mas a mortalidade é superior, tais como entre indígenas australianos de New South Wales em que a incidência foi $18 \%$ menor e a mortalidade $86 \%$ maior ${ }^{32}$. Sugere-se que nesses países a menor triagem com o teste de PSA (Antígeno prostático específico) em indígenas do que entre não indígenas influencie na menor incidência, enquanto a maior mortalidade pode ser devido a disparidades no acesso ao diagnóstico precoce e tratamento oportuno 65,66 .

Em relação ao câncer de mama, configura-se como uma das principais causas de óbitos por neoplasias em mulheres no mundo e nos países em desenvolvimento 28. Em 2015, ocupou o primeiro lugar nas regiões Sudeste, Sul, Centro-oeste (exceto Goiás) e Nordeste (exceto Piauí e Maranhão) do Brasil 30. Entre 1990 e 2015, exibiu estabilidade, sendo verificada uma tendência de aumento com destaque para o Acre, que pode ser relacionada ao envelhecimento populacional, ampliação da exposição a fatores de risco e menor disponibilidade de detecção precoce com tratamento oportuno 19,30. No presente estudo, identificou-se menor mortalidade entre indígenas em relação a todas as populações de referência, resultados coerentes com a análise que apontou menor risco de óbito por câncer de mama entre mulheres indígenas brasileiras quando comparadas a brancas em 2000 ( $R R=0,25$; IC95\%: 0,13-0,47) e 2010 ( $R R=0,09$; IC95\%: 0,03-0,22) 67. Em países desenvolvidos, embora a taxa de incidência por câncer de mama entre mulheres indígenas seja inferior à identificada entre mulheres não indígenas, observa-se um ritmo elevado de óbitos em indígenas, sendo verificada detecção tardia, estadiamento avançado, menor participação no rastreamento e pior sobrevida como reflexo das disparidades de acesso ao tratamento 68,69 .

No Brasil, estudos demográficos realizados em etnias específicas têm demonstrado o aparecimento do câncer no conjunto das causas de óbitos, tais como entre os índios Kaingáng no Rio Grande do Sul, que entre 1985 e 1995 registraram quatro óbitos por câncer de colo uterino, e entre os Suyá, no Parque Indígena do Xingu, que entre 1970 e 2004 registraram dois óbitos por esta mesma neoplasia, um por câncer de cólon, endométrio e linfoma não-Hodgkin, sendo que entre 1990 e 2004 as neoplasias ocuparam a segunda posição de mortalidade $(21,4 \%)$ 15,16. Na população indígena Pakaánova de Guajará Mirim, Rondônia, entre 1995 e 1997, ocorreram dois óbitos por câncer (leucemia e o outro não informado) 17. Entre os Xavante, as neoplasias corresponderam a 10,8\% das causas de mortes observadas entre 1999 e 2002, com registros de óbitos por cânceres de localização uterina, hepática, renal e leucemia, todos em mulheres de 30-49 anos 18 .

Os povos indígenas detêm um elevado pluralismo cultural e enfrentam inúmeras barreiras à saúde, para além do isolamento físico, tais como as relacionadas à organização dos serviços de saúde, dificuldades de referência a atendimentos especializados, ausência de material educativo culturalmente apropriado e desconsideração da perspectiva étnica no processo terapêutico 47,70 . Em relação à população do Acre e Região Norte, embora a oferta de ações de saúde esteja disponível para a população geral e indígena, as barreiras ligadas aos idiomas, culturas, modos de vida indígena e organização dos serviços podem afetar o acesso, o estadiamento, a adesão ao rastreio e ao tratamento das neoplasias. Do mesmo modo, em relação a Goiânia, o acesso às informações, serviços de rastreio, 
diagnóstico e tratamento podem favorecer à menor mortalidade por câncer nessa população do que entre indígenas 27.

Entre as limitações do estudo, apontamos que embora o registro de óbitos no Brasil tenha apresentado melhora na qualidade desde o ano 2000, a Região Norte ainda apresenta percentual de óbitos por causa mal definida (CMD) acima da meta (< 10\%). No Acre, esse percentual foi de 19,1\% em 201071. Nas populações indígenas, entre 2000 e 2012, o percentual foi de aproximadamente 15\%. Todavia, em nossas análises, optamos por adotar uma postura mais conservadora e não reclassificamos os óbitos por causas mal definidas, utilizando para o cálculo da RMP apenas o número de óbitos reais (observados). Contudo, mesmo sem utilizarmos tais dados, encontramos excessos entre indígenas se comparados às populações de referência.

A qualidade das informações do SIM depende do correto preenchimento das declarações de óbito, refletindo também a capacidade diagnóstica dos serviços. Todavia, dados de morte por câncer são bastante fidedignos. Em relação à categoria raça/cor existem dificuldades para mensuração, desfavorecida pela impossibilidade da autoclassificação, que pode contribuir com o sub-registro em indígenas 72. Embora tenhamos acessado o banco nominal para ampliar a identificação indígena, reconhecemos que muitos indivíduos podem não usar a designação étnica nos sobrenomes, inviabilizando a reclassificação de indígenas não categorizados como tal no item raça/cor. Outras limitações estão relacionadas às diferenças dos critérios de classificação indígena dos censos de 2000 e 2010, bem como às estimativas dos IC95\% das RMP para neoplasias de próstata, colorretal e leucemias em homens, e estômago, fígado, leucemia e mama entre mulheres, que apresentaram menos de seis óbitos, podendo não atender ao pressuposto de distribuição aproximadamente normal.

No nosso conhecimento, o presente estudo é o primeiro da literatura nacional a fornecer estimativas de base populacional de mortalidade por câncer entre indígenas, na dimensão estadual, proporcionando conhecimento sobre o tema entre povos oriundos da Região Amazônica. Considerando que o RCBP no Acre ainda está em fase de implantação, as estimativas podem contribuir para a compreensão do quadro epidemiológico das neoplasias entre indígenas e no estabelecimento de políticas públicas para esses povos.

Nossos achados destacam a relevância de neoplasias passíveis de prevenção, como de colo uterino, e aquelas ligadas ao subdesenvolvimento, como estômago e fígado, no quadro de mortalidade por neoplasias entre indígenas, com magnitudes superiores ao esperado quando comparadas às populações de referência. Esses achados apontam para a necessidade de estruturação dos programas de controle do câncer cervical, além do diagnóstico e tratamento oportuno para o conjunto das neoplasias observadas. Ressalta-se a importância da adequação dos serviços às necessidades das populações indígenas, envolvendo programas de educação em saúde relativos ao rastreamento, diagnóstico e tratamento oncológico, culturalmente sensíveis e em trabalho de parceria com as comunidades.

\section{Colaboradores}

M. F. S. O. Borges contribuiu na concepção do projeto, coleta de dados, análise e interpretação dos dados, redação e revisão do artigo, e aprovação da versão final. R. J. Koifman contribuiu na concepção do projeto, análise e interpretação dos dados, redação e revisão do artigo, e aprovação da versão final. I. F. Silva contribuiu na concepção do projeto, análise e interpretação dos dados, redação e revisão do artigo, e aprovação da versão final.

\section{Informações adicionais}

ORCID: Maria Fernanda de Sousa Oliveira Borges (0000-0002-5536-6507); Rosalina Jorge Koifman (0000-0002-2746-7597); Ilce Ferreira da Silva (0000-0002-7134-3030).

\section{Agradecimentos}

À Fundação de Amparo à Pesquisa do Acre (FAPAC). 


\section{Referências}

1. Anderson I, Robson B, Connolly M, Al-Yaman F, Bjertness E, King A, et al. Indigenous and tribal people" health (The Lancet-Lowitja Institute Global Collaboration): a population study. Lancet 2016; 388:131-57.

2. Instituto Brasileiro de Geografia e Estatística. Características gerais dos indígenas no Censo Demográfico 2010: resultados do Universo. Rio de Janeiro: Instituto Brasileiro de Geografia e Estatística; 2012.

3. Braga WSM, Brasil LM, Souza RAB, Castilho $\mathrm{MC}$, Fonseca JC. Ocorrência da infecção pelo vírus da hepatite $\mathrm{B}$ (VHB) e delta (VHD) em sete grupos indígenas do Estado do Amazonas. Rev Soc Bras Med Trop 2001; 34:349-55.

4. Orellana JDY, Basta PC, Santos RV, Coimbra Jr., CEA. Morbidade hospitalar em crianças indígenas Suruí menores de dez anos, Rondônia, Brasil: 2000 a 2004. Rev Bras Saúde Mater Infant 2007; 7:281-7.

5. Rios DPG, Malacarne J, Alves LCC, Sant'Anna CC, Camacho LAB, Basta PC. Tuberculose em indígenas da Amazônia brasileira: estudo epidemiológico na Região do Alto Rio Negro. Rev Panam Salud Pública 2013; 33:22-9.

6. Coimbra Jr. CE, Santos RV, Welch JR, Cardoso AM, Souza MC, Garnelo L, et al. The First National Survey of Indigenous People's Health and Nutrition in Brazil: rationale, methodology, and overview of results. BMC Public Health 2013; 13:52.

7. Lapouble OMM, Santelli, ACFS, Muniz-Junqueira MI. Situação epidemiológica da malária na Região Amazônica Brasileira, 2003 a 2012. Rev Panam Salud Pública 2015; 38:300-6.

8. Araújo TS, Oliveira CSM, Muniz PT, Silva-Nunes M, Cardoso MA. Desnutrição infantil em um dos municípios de maior risco nutricional do Brasil: estudo de base populacional na Amazônia Ocidental Brasileira. Rev Bras Epidemiol 2016; 19:554-66.

9. Oliveira RA, Gurgel-Gonçalves R, Machado ER. Intestinal parasites in two indigenous ethnic groups in northwestern Amazonia. Acta Amaz 2016; 46:241-6.

10. Oliveira GFO, Rodrigues TRR, França F, Corrêa LF, Ikejiri AT, Casulari LA. Prevalência de diabetes melito e tolerância à glicose diminuída nos indígenas da Aldeia Jaguapiru, Brasil. Rev Panam Salud Pública 2011; 29:315-21.

11. Rocha AKS, Bós AJG, Huttner E, Machado DC. Prevalência da síndrome metabólica em indígenas com mais de 40 anos no Rio Grande do Sul, Brasil. Rev Panam Salud Pública 2011; 29:41-5.

12. Souza-Filho ZA, Ferreira AA, Santos BD, Pierin AM. Hypertension prevalence among indigenous populations in Brazil: a systematic review with meta-analysis. Rev Esc Enferm USP 2015; 49:1016-26.

13. Aguiar Jr. PN, Stock GT, Lopes Jr. GL, Tadokoro H, Gutierres BS, et al. Disparidades na epidemiologia e no tratamento de câncer nas populações indígenas brasileiras. Einstein (São Paulo) 2016; 14:330-7.
14. Campos MB, Borges GM, Queiroz BL, Santos RV. Diferenciais de mortalidade entre indígenas e não indígenas no Brasil com base no Censo Demográfico de 2010. Cad Saúde Pública 2017; 33:e00015017.

15. Hökerberg YHM, Duchiade MP, Barcellos C. Organização e qualidade da assistência à saúde dos índios Kaingángs do Rio Grande do Sul, Brasil. Cad Saúde Pública 2001; 17:261-72.

16. Pagliaro H, Carvalho NS, Rodrigues D, Baruzzi RG. Demographic dynamics of the Suyá, a Jê people of the Xingu Indigenous Park, Central Brazil, 1970-2004. Cad Saúde Pública 2007; 23:1071-81.

17. Escobar AL, Coimbra Jr. CEA. Considerações sobre as Condições de Saúde das Populações das Áreas Indígenas Pakaánova (Wari) e do Posto Indígena Cuaporé, Rondônia. Documento de Trabalho no 1. Porto Velho: Centro de Estudos em Saúde do Índio de Rondônia, Universidade Federal de Rondônia; 1998.

18. Souza LG, Santos RV, Coimbra Jr. CEA. Estrutura etária, natalidade e mortalidade do povo indígena Xavante de Mato Grosso, Amazônia, Brasil. Ciênc Saúde Colet 2010; 15 Suppl 1:1465-73.

19. Nakashima JP, Koifman S, Koifman RJ. Tendência da mortalidade por neoplasias malignas selecionadas em Rio Branco, Acre, Brasil, 19802006. Cad Saúde Pública 2011; 27:1165-74.

20. Escobar AL, Rodrigues AF, Alves CLM, Orellana JDY, Santos RV, Coimbra Jr. CEA. Causas de internação hospitalar indígena em Rondônia. O Distrito Sanitário Especial Indígena Porto Velho (1998-2001). In: Coimbra Jr. CEA, Santos RS, Escobar AL, organizadores. Epidemiologia e saúde dos povos indígenas no Brasil. Rio de Janeiro: Editora Fiocruz/ABRASCO; 2003; p. 127-47.

21. Lunardi R, Santos RV, Coimbra Jr CEA. Morbidade hospitalar de indígenas Xavante, Mato Grosso, Brasil (2000-2002). Rev Bras Epidemiol 2007; 10:441-52.

22. Rothman KJ, Greenland S, Timothy LL. Epidemiologia moderna. 3a Ed. Porto Alegre: Artmed; 2011.

23. Moura L, Curado MP, Simôes EJ, Cezário AC, Urdaneta M. Avaliação do registro de câncer de base populacional do Município de Goiânia, Estado de Goiás, Brasil. Epidemiol Serv Saúde 2006; 15:7-17.

24. Bray F, Colombet M, Mery L, Piñeros M, Znaor A, Zanetti R, et al. Cancer incidence in five continents. v. XI. Lyon: International Agency for Research on Cancer; 2017.

25. Frias PG, Szwarcwald CL, Lira PIC. Avaliação dos sistemas de informações sobre nascidos vivos e óbitos no Brasil na década de 2000. Cad Saúde Pública 2014; 30:2068-80.

26. Kahn HA, Sempos CT. Statistical methods in epidemiology. 2nd Ed. New York: Oxford University Press; 1989. 
27. Goss PE, Lee BL, Badovinac-Crnjevic T, Strasser-Weippl K, Chavarri-Guerra Y, St Louis J, et al. Planning cancer control in Latin America and the Caribbean. Lancet Oncol 2013; 14:391-436.

28. Ferlay J, Soerjomataram I, Ervik N, Dikshit R, Eser S, Mathers C, et al. GLOBOCAN 2012: estimated cancer incidence, mortality and prevalence worldwide in 2012. v 1.0. Lyon: International Agency for Research on Cancer; 2013. (IARC CancerBase, 11).

29. Coordenação de Prevenção e Vigilância, Instituto Nacional de Câncer José Alencar Gomes da Silva. Estimativa 2016: incidência de câncer no Brasil. Rio de Janeiro: Instituto Nacional de Câncer José Alencar Gomes da Silva; 2015.

30. Guerra MR, Bustamante-Teixeira MT, Corrêa CSL, Abreu DMX, Curado MP, Mooney M, et al. Magnitude e variação da carga da mortalidade por câncer no Brasil e Unidades da Federação, 1990 e 2015. Rev Bras Epidemiol 2017; 20 Suppl 1:102-15.

31. Espey D, Paisano R, Cobb N. Regional patterns and trends in cancer mortality among American Indians and Alaska Natives, 1990-2001. Cancer 2005; 103:1045-53.

32. Morrell S, You H, Baker D. Estimates of cancer incidence, mortality and survival in aboriginal people from NSW, Australia. BMC Cancer 2012; 12:168.

33. Resende ALS, Mattos IE, Koifman S. Mortalidade por câncer gástrico no Estado do Pará, 1980-1997. Arq Gastroenterol 2006; 43:24752.

34. Plummer M, Franceschi S, Vignat J, Forman D, Martel C. Global burden of gastric cancer attributable to pylori. Int J Cancer 2015; 136:487-90

35. Escobar-Pardo ML, Godoy APO, Machado RS, Rodrigues D, Fagundes Neto U, Kawakami E. Prevalência da infecção por Helicobacter pylori e de parasitoses intestinais em crianças do Parque Indígena do Xingu. J Pediatr (Rio J.) 2011; 87:393-8.

36. Roque JRS, Machado RS, Rodrigues D, Rech P, Kawakami E. Prevalência de infecção por Helicobacter pylori em uma comunidade indígena em São Paulo e fatores associados: estudo transversal. São Paulo Med J 2017; 135:140-5.

37. Arnold M, Moore SP, Hassler S, Ellison-Loschmann L, Forman D, Bray F. The burden of stomach cancer in indigenous populations: a systematic review and global assessment. Gut 2014; 63:64-71.

38. Ferlay J, Soerjomataram I, Dikshit R, Eser S, Mathers C, Rebelo M, et al. Cancer incidence and mortality worldwide: sources, methods and major patterns in GLOBOCAN 2012. Int J Cancer 2015; 136:E359-86.

39. Barbosa IR, Souza DLB, Bernal MM, Costa ICC. Desigualdades regionais na mortalidade por câncer de colo de útero no Brasil: tendências e projeções até o ano 2030. Ciênc Saúde Colet 2016; 21:253-62.
40. O’Brien ED, Bailie RS, Jelfs PL. Cervical cancer mortality in Australia: contrasting risk by aboriginality, age and rurality. Int J Epidemiol 2000; 29:813-6.

41. Moore SP, O’Rourke PK, Mallitt KA, Garvey G, Green AC, Coory MD, et al. Cancer incidence and mortality in Indigenous Australians in Queensland, 1997-2006. Med J Aust 2010; 193:590-3

42. Fonseca ALDC, Amorim LDC, Murari RSW, Arcoverde LC, Ferreira LCL. Prevalência de alterações citológicas cervicais em indígenas do Extremo Norte da Amazônia Brasileira. Rev Bras Cancerol 2014; 60:101-8.

43. Solé Pla MA, Corrêa FM, Claro IB, Silva MAF, Kneipp MB, Bortolon PC. Análise descritiva do perfil dos exames citopatológicos do colo do útero realizados em mulheres indígenas e não indígenas no Brasil, 2008-2011. Rev Bras Cancerol 2012; 58:461-69.

44. Glick SB, Clarke AR, Blanchard A, Whitaker AK. Cervical cancer screening, diagnosis and treatment interventions for racial and ethnic minorities: a systematic review. J Gen Intern Med 2012; 27:1016-32.

45. Hill S, Sarfati D, Robson B, Blakely T. Indigenous inequalities in cancer: what role for health care? ANZ J Surg 2013; 83:36-41.

46. Speck NMG, Pinheiro JS, Pereira ER, Rodrigues D, Focchi GRA, Ribalta JCL. Rastreamento do câncer de colo uterino em jovens e idosas do Parque Indígena do Xingu: avaliação quanto à faixa etária preconizada no Brasil Einstein (São Paulo) 2015; 13:52-7.

47. Stephens C, Nettleton C, Porter J, Willis R, Clark S. Indigenous peoples' health: why are they behind everyone, everywhere? Lancet 2005; 366:10-3.

48. Torre LA, Bray F, Siegel RL, Ferlay J, LortetTieulent J, Jemal A. Global cancer statistics, 2012. CA Cancer J Clin 2015; 65:87-108.

49. Amorim TR, Merchán-Hamann E. Mortalidade por neoplasia maligna do fígado e vias biliares intra-hepáticas no Brasil, 1980-2010. Cad Saúde Pública 2013; 29:1427-36.

50. Paula VS, Arruda ME, Vitral CL, Gaspar AMC. Seroprevalence of viral hepatitis in riverine communities from the Western Region of the Brazilian Amazon Basin. Mem Inst Oswaldo Cruz 2001; 96:1123-8.

51. Viana S, Paraná R, Moreira RC, Compri AP, Macedo V. High prevalence of hepatitis $b$ and hepatitis D virus in the western Brazilian Amazon. Am J Trop Med Hyg 2005; 74:808-14.

52. Koifman S, Ferraz I, Viana TS, Silveira CLP, Carneiro MTD, Koifman RJ, et al. Cancer cluster among young Indian adults living near power transmission lines in Bom Jesus do Tocantins, Pará, Brazil. Cad Saúde Pública 1998; 14 Suppl 3:S161-72.

53. San Sebastian M, Armstrong B, Stephens C. Environmental exposure and cancer incidence near oil fields in the Amazon basin of Ecuador. Occup Environ Med 2001; 58:517-22. 
54. Ishak R, Vallinoto ACR, Azevedo VN, Ishak MQ. Epidemiological aspects of retrovirus (HTLV) infection among Indian populations in the Amazon Region of Brazil. Cad Saúde Pública 2003; 19:109-14.

55. Departamento de Atenção Básica, Secretaria de Atenção à Saúde, Ministério da Saúde. Rastreamento. Brasília: Ministério da Saúde: 2010. (Série A. Normas e Manuais Técnicos) (Cadernos de Atenção Primária, 29).

56. Johnson CM, Wei C, Ensor JE, Smolenski DJ, Amos CI, Levin B. et al. Meta-analyses of colorectal cancer risk factors. Cancer Causes Control 2013; 24:1207-22.

57. Cardoso AM, Santos RV, Garnelo L, Coimbra Junior CEA, Chaves MBG. Políticas públicas de saúde para os povos indígenas. In: Giovanella L, Lobato LVC, Noronha JC, Carvalho AI, organizadores. Políticas e sistema de saúde no Brasil. 2a Ed. Rio de Janeiro: Editora Fiocruz; 2012. p. 911-32.

58. Marrett LD, Chaudhry M. Cancer incidence and mortality in Ontario First Nations, 19681991 (Canada). Cancer Causes Control 2003; 14:259-68.

59. Louchini R, Beaupré M. Cancer incidence and mortality among Aboriginal people living on reserves and northern villages in Québec, 1988-2004. Int J Circumpolar Health 2008; 67:445-51

60. Perdue DG, Haverkamp D, Perkins C, Daley CM, Provost E. Geographic variation in colorectal cancer incidence and mortality, age of onset, and stage at diagnosis among American Indian and Alaska Native People, 19902009. Am J Public Health 2014; 104 Suppl 3:S404-14.

61. White MC, Espey DC, Swan J, Wiggins CL, Eheman C, Kaur JS, Disparities in cancer mortality and incidence among American Indians and Alaska Natives in the United States. Am J Public Health 2014; 104 Suppl 3:S377-87.

62. Condon JR, Barnes T, Armstrong BK, Nayagam SS, Elwood M. Stage at diagnosis and cancer survival for Indigenous Australians in the Northern Territory. Med J Aust 2005; 182:277-80

63. Hill S1, Sarfati D, Blakely T, Robson B, Purdie G, Chen J, et al. Survival disparities in Indigenous and non-Indigenous New Zealanders with colon cancer: the role of patient comorbidity, treatment and health service factors. J Epidemiol Community Health 2010; 64:117-23.
64. Weir K, Supramaniam R, Gibberd A, Dillon A, Armstrong BK, O'Connell DL. Comparing colorectal cancer treatment and survival for aboriginal and non-aboriginal people in New South Wales. Med J Aust 2016; 204:156.

65. Moore SP, Antoni S, Colquhoun A, Healy B, Ellison-Loschmann L, Potter JD, et al. Cancer incidence in indigenous people in Australia, New Zealand, Canada, and the USA: a comparative population-based study. Lancet Oncol 2015; 16:1483-92.

66. Rodger JC, Supramaniam R, Gibberd AJ, Smith DP, Armstrong BK, Dillon A, et al. Prostate cancer mortality outcomes and patterns of primary treatment for Aboriginal men in New South Wales, Australia. BJU Int 2015; 115 Suppl 5:16-23.

67. Freitas-Junior R, Soares LR, Gonzaga CMR, Sousa ALL, Lima MG, Branquinho LW, et al. Mortalidade por câncer de mama em mulheres indígenas brasileiras. Rev Bras Mastologia 2015; 25:41-5.

68. Ahmed S, Shahid RK, Episkenew JA. Disparity in cancer prevention and screening in aboriginal populations: recommendations for action. Curr Oncol 2015; 22:417-26.

69. Tapia KA, Garvey G, Mc Entee M, Rickard M, Brennan P. Breast cancer in Australian indigenous women: incidence, mortality, and risk factors. Asian Pac J Cancer Prev 2017; 18:87384.

70. Pontes ALM, Garnelo L, Rego S. Reflexões sobre questões morais na relação de indígenas com os serviços de saúde. Rev Bioét (Impr.) 2014; $22: 337-46$

71. Cunha CC, Teixeira R, França E. Avaliação da investigação de óbitos por causas mal definidas no Brasil em 2010. Epidemiol Serv Saúde 2017; 26:19-30.

72. Cardoso AM, Santos RV, Coimbra Jr. CEA. Mortalidade infantil segundo raça/cor no Brasil: o que dizem os sistemas nacionais de informação? Cad Saúde Pública 2005; 21:1602-8. 


\section{Abstract}

The study aimed to estimate cancer mortality among indigenous peoples in Acre State, Brazil. This was a descriptive observational study based on the nominal bank of the Brazilian Mortality Information System for the period from January 1st, 2000, to December 31st, 2012. The study analyzed the distribution death frequencies by sex and age. Standardized mortality ratio (SMR) was calculated taking Goiânia (Goiás State), Acre State, and the North Region of Brazil as the references. A total of 81 deaths were identified, the majority in men (59.3\%) and in individuals over 70 years of age. The five main sites in men were stomach, liver, colon and rectum, leukemia, and prostate. The five main sites in women were uterine cervix, stomach, liver, leukemia, and uterus. In indigenous men there was an excess of deaths from stomach cancer compared to the populations of Goiânia $(S M R=2.72 ; 2.58-2.87)$, Acre State $(S M R=$ 2.05 ; 1.94-2.16) and North region $(S M R=3.10$; 2.93-3.27). The same was observed for deaths from hepatic cell carcinomas referenced against Goiânia (SMR = 3.89; 3.66-4.14), Acre State $(S M R=1.79 ; 1.68-1.91)$, and the North of Brazil (SMR = 4.04; 3.77-4.30). Among indigenous women, there was an excess of cervical cancer in comparison to Goiânia (SMR = 4.67; 4.41-4.93), Acre State $(S M R=2.12 ; 2.00-2.24)$, and the North $(S M R=2.60 ; 2.45-2.75)$. The estimates show that preventable neoplasms such as cervical cancer and those linked to underdevelopment, such as stomach and liver cancer, account for $49.4 \%$ of deaths among indigenous peoples. Compared to the reference population, mortality from liver, stomach, and colorectal cancer and leukemias was more than twice as high in indigenous men; among indigenous women, cervical, stomach, and liver cancer and leukemias were $30 \%$ higher.

Health of Indigenous Peoples; Neoplasms; Mortality

\section{Resumen}

El objetivo del estudio fue estimar la mortalidad por cáncer en pueblos indígenas del estado de Acre. Se trata de un estudio observacional descriptivo, a partir del banco de datos nominal del SIM (Sistema de Información Sobre Mortalidad), referente al período de 01 de enero de 2000 a 31 de diciembre de 2012. Se analizó la distribución de frecuencia de óbitos, por sexo y franja de edad, y se calculó la RMP (razón de mortalidad estandarizada), teniendo como referencia Goiânia-GO, Acre y la región norte. Se identificaron 81 óbitos, la mayoría en hombres $(59,3 \%)$ y por encima de los 70 años. Los cinco principales focos en hombres fueron: estómago, hígado, colon y recto, leucemia y próstata. En las mujeres, cáncer cervical, estómago, hígado, leucemia y útero. En los hombres indígenas, hubo exceso de óbitos por cáncer de estómago, comparados con las poblaciones de Goiânia ( $R M P=2,72$; 2,58-2,87), estado de Acre $(R M P=2,05 ; 1,94$ 2,16) y región norte $(R M P=3,10 ; 2,93-3,27)$. Lo mismo se observó en caso de óbitos por hepatocarcinomas, teniendo por referencia Goiânia $(R M P=$ 3, 89; 3,66-4,14), estado de Acre ( $R M P=1,79$; $1,68-1,91)$ y región norte $(R M P=4,04 ; 3,77-4,30)$. Entre las mujeres indígenas, se destaca el exceso de cáncer cervical, en relación con Goiânia $(R M P=$ 4,67; 4,41-4,93), estado de Acre $(R M P=2,12$; $2,00-2,24)$ y región norte $(R M P=2,60 ; 2,45-2,75)$. Las estimativas apuntan a que neoplasias susceptibles de prevención, como la cervical, y vinculadas al subdesarrollo, como la de estómago e hígado, correspondieron a cerca de un 49,4\% de los óbitos entre indígenas. Comparados con la población de referencia, la mortalidad por cáncer de hígado, estómago, colorrectal y leucemias fue más que el doble entre los hombres indígenas; por cáncer cervical, estómago, hígado y leucemias estuvo por encima de un 30\% entre las mujeres indígenas.

Salud de Poblaciones Indígenas; Neoplasias; Mortalidad
Recebido em 20/Jul/2018

Versão final reapresentada em 03/Jan/2019 Aprovado em 24/Jan/2019 